\title{
EVALUATION OF SINUS FLOOR AUGMENTATION WITH AND WITHOUT PLATELET -RICH FIBRIN WITH SIMULTANEOUS IMPLANT PLACEMENT
}

\author{
Shaimaa S. Nasr*, Mohamed Sherien ${ }^{* *}$ and Amr Zahran**
}

\begin{abstract}
Introduction: Implant dentistry had widened the prosthetic possibilities; therefore they became commonly used in many ways for substitution of lost teeth. However, there may be contraindications for the placement of dental implants. presence of sinus pneumatization in the posterior maxilla is a frequent anatomical obstacle; occurs as a result of gradual thinning of sinus walls with aging and reduction of maxillary masticatory forces. preparation of buccal window to gain access to the sinus cavity followed by elevation of the Schniederian membrane to create secluded compartment for augmentation material and /or implant placement. PRF can be used as a sole filling material during a simultaneous sinus lift and implantation stabilized a high volume of natural regenerated bone in the subsinus cavity up to the tip of the implants; and considered a satisfactory alternative.
\end{abstract}

Materials and methods: Twenty medically free patients (both males and females) received sinus floor augmentation with simultaneous implant placement. The patients were divided into two groups; where first group received platelet rich fibrin (PRF) as filling material with PRF membrane; while the other group received xenograft with collagen membrane.

Results: When comparing the L-PRF group with xenograft group after 5 months, it was found that mean crestal bone loss in L-PRF group was less than that in xenograft group.

Conclusion: L-PRF can be used successively as a sinus grafting material and as a membrane.

KEYWORDS: Sinus floor augmentation, platelet rich fibrin, bone grafts, collagen membrane, guided bone regeneration.

\section{INTRODUCTION}

Presence of sinus pneumatization in the posterior maxilla is a frequent anatomical obstacle; occurs as a result of gradual thinning of sinus walls with aging and reduction of maxillary masticatory forces (Chanavaz 1990). Therefore, Prosthetic rehabilitation of the edentulous maxilla using endosteal implants will be limited by the insufficient quality and quantity of available bone 5 . Sinus floor augmentation is considered a valuable approach to

\footnotetext{
* Assistant Lecturer, Oral Medicine and Periodontology Department, Faculty of Oral and Dental Medicine, Fayoum University.
}

***Professor of Oral Medicine and Periodontology Department, Faculty of Oral and Dental Medicine, Cairo University. 
increase the absolute bone height in the maxilla; to provide sufficient anchorage for implant placement (Tatum 1986).

\section{Different modalities for sinus floor augmentation}

Sinus floor augmentation can be performed via two approaches, internal or external according to the available subsinus height. Internal approach; is considered as minimal invasive procedure for sinus lifting and indicated when the subsinus height is equal to $6 \mathrm{~mm}$ or more. It was first suggested by Tatum 1986; where a socket former for the selected implant site was used and green stick fracture of the sinus floor was accomplished by hand tapping the socket former in a vertical direction. After preparation of the implant site, the implant was placed and allowed to heal in a submerged manner (Tatum et al., 1986). Summers 1994, later on described the osteotome technique for sinus floor elevation; with set of osteotomes of different diameters aiming for increasing the density of the soft type III and IV maxillary bone to increase the primary stability of the implant.

\section{Lateral approach for sinus floor augmentation}

This technique was first introduced by Tatum 1986 and modified by(Boyne \& James 1980), and (Wood \& Moore 1988); which involves preparation of buccal window to gain access to the sinus cavity followed by elevation of the Schniederian membrane to create secluded compartment for augmentation material and /or implant placement. It is considered one of the most predictable alternatives for sinus floor augmentation especially in severely resorbed alveolar bone; it allows direct and adequate view of the sinus, enabling greater amount of augmentation material to be placed in the appropriate position and packed to the maximum (Peleg et al. 2006).

Noteworthy, lateral approach in sinus floor augmentation can be performed via single stage with simultaneous implant placement, and this is recommended when there is a minimum of $5 \mathrm{~mm}$ residual bone height to ensure adequate primary implant stability and parallelism. Additionally, simultaneous implant placement creates tenting effect resulting in excellent space maintenance for optimal regeneration. (Lundgren et al. 2004).

Different bone substitutes used with sinus floor augmentation; such as Autogenous grafts which are harvested from the patient's own body. It has osteoconductive and osteoinductive and osteogenic properties because it contains source of osteoprogenitor cells (Rosenberg \& Rose 1998). It can be taken from intraoral site like maxillary tuberosity, mental or retromolar area, or edentulous alveolar area; and also can be taken from extraoral sites as iliac crest or tibia (Nasr et al. 1999).

Allografts are obtained from other individuals of the same species but of disparate genotype. Bone allografts are the most frequently used alternative to autogenous grafts. They include both freeze-dried bone allografts (FDBA) and demineralized freeze dried bone allografts (DFDBA). They are provided from approved tissue banks as and are and treated and manipulated through different methods and steps of cleansing, decontamination, microbiological treatment, freezing, lyophilization, packaging, and sterilization - to provide them free of contaminants and diminish the risk of disease transfer (Holtzclaw et al. 2008, Reynolds et al. 2010). In addition to Alloplasts (synthetic grafts): These are synthetic biocompatible inorganic grafting material such as ceramics and polymers.

Absorbable gelatin sponge is another grafting material in sinus lifting; where a study by (Sohn et al. 2010)concluded that placement of a dental implant in the maxillary sinus with a gelatin sponge can be a predictable procedure for sinus augmentation.

Xenografts: These grafts are de-protenized cancellous skeletal bone tissue that is harvested from genetically different species and transferred to the recipient site of another species. Several studies conducted on xenografts showed good clinical 
results when used for augmentation of the alveolar crest or maxillary sinus for implant placement (Wenz et al. 2001, Ning et al. 2009).

Platelet -rich fibrin (PRF) plug: Plateletrich- fibrin (PRF), a second generation platelet concentrate first described by Choukron (Mazor et al. 2009). Its essence is a fibrin matrix in which the platelets, cytokines and cells are trapped and may be released after a certain time (MW 2005). These cytokines had been shown to stimulate the mitogenic response of the periosteum during the early stage of bone repair (Gruber et al. 2003) and in general are strongly associated with the bone healing process (Metzler et al. 2012).

Moreover, L-PRF releases high amounts of growth factors (such as transforming growth factor- $\beta 1$ [TGF $\beta-1]$, platelet-derived growth factor$\mathrm{AB}$ [PDGF-AB], and matrix glycoproteins (such as thrombospondin-1) during at least 7 days in vitro (Dohan Ehrenfest, de Peppo, et al. 2009). In addition to vascular endothelial growth factor [VEGF]), which is a potent angiogenic growth factor, having direct chemotactic and mitogenic effects on osteoblasts and osteogenic cells. Thus, it could have direct and indirect effects on bone regeneration via stimulating many different kinds of cells, particularly the proliferation and differentiation of osteoblasts as part of GBR procedures (Kaigler et al. 2013). Therefore, use of L-PRF during sinuslift procedures had been advocated for many years during lateral sinus-lifting or vertical osteotome augmentation (Diss et al. 2008). Therefore, the aim of the study is to evaluate sinus floor augmentation with platelet rich fibrin versus xenograft and membrane with simultaneous implant placement.

\section{MATERIALS AND METHODS}

\section{I) Patients Selection}

This study was conducted on twenty patients (both males and females) with age range between 37-50 years old. The selected patients were requiring single or multiple implant placements in the maxillary posterior region, admitted to the Outpatient Clinic of Oral Medicine, Diagnosis and Periodontology department, Faculty of oral and dental medicine, Cairo University. Patients were medically free and not receiving any medication for at least 6 months prior to the surgery to avoid interference of any external factor. Absence of any pathological condition in the recipient site. Residual bone height from the alveolar crest to the maxillary sinus floor about $4-5 \mathrm{~mm}$. Adequate ridge width was $(\geq 6 \mathrm{~mm})$. Adequate interarch space was $(\geq 5 \mathrm{~mm})$. Patients were cooperative, highly motivated and with good oral hygiene status.

\section{II) Presurgical evaluation}

Panoramic radiographs were taken for all patients to detect; the residual bone height (from the crest of the alveolar ridge up to the maxillary sinus floor), or absence of any clinically undetectable pathology. In addition to CBVT was performed for all patients before any procedure, immediately after the surgery, and after 5 months follow up.

\section{III) Preoperative impression}

Impressions were taken for each patient with alginate impression to obtain study casts; to show the interarch space adequacy and to detect if there is any occlusal discrepancy.

\section{IV) Surgical protocol}

Surgery was performed with local infiltration anaesthesia. Then, access to buccal maxillary bone was performed via mucosal crestal incision; with anterior and posterior releasing vestibular incisions. Then, full thickness flap was reflected to expose the lateral wall of the maxilla and portion of the zygoma, as shown in figure (1). 


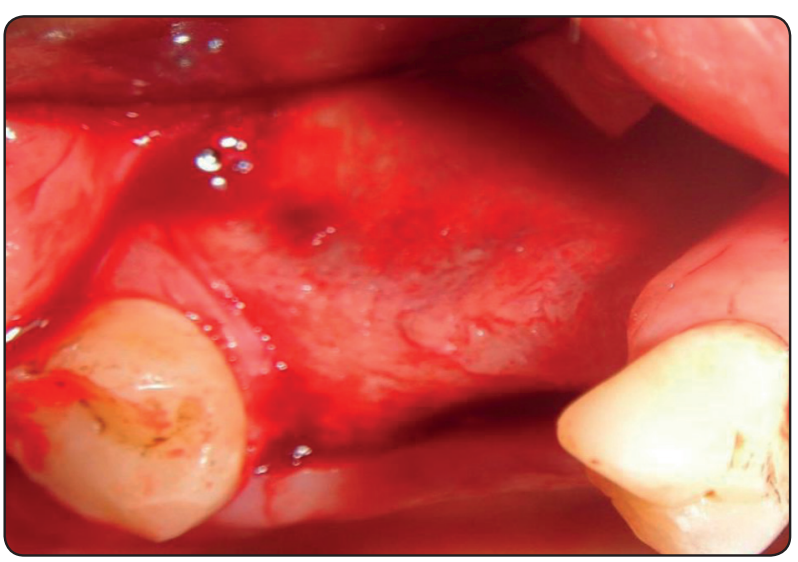

Fig. (1) Showing flap reflection in one of the cases.

Then, a bone window was outlined using piezoelectric device* using piezoelectric tip SG7D, with constant saline irrigation. The size of the window was dependent on the number of implants required, as shown in figure Gently the lateral access window was infractured from the surrounding bone then, with gentle release of the membrane from the surrounding walls of the sinus with piezoelectric tip SG11, as shown in figure (2).

Careful elevation of the Schneiderian membrane with curved elevator was performed; to increase the available subsinus cavity that was grafted for implant placement, as shown in figure (3).

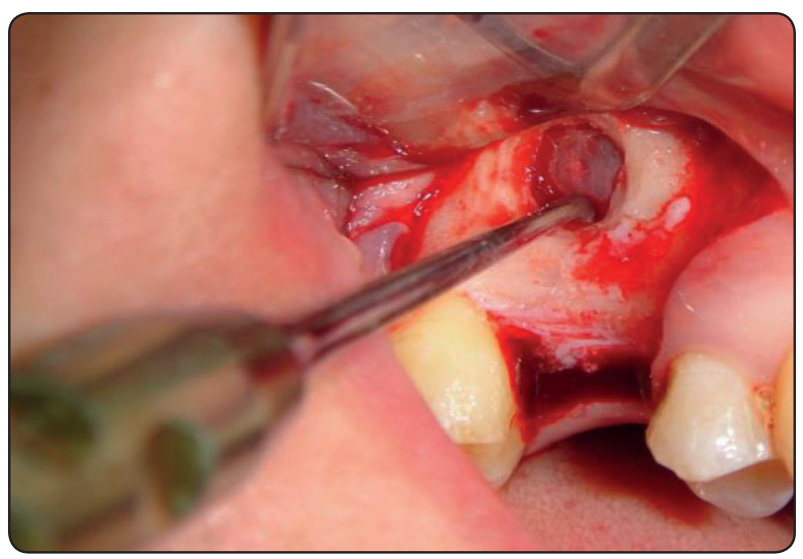

Fig. (3) Showing elevating the Schneiderian membrane with curved elevator

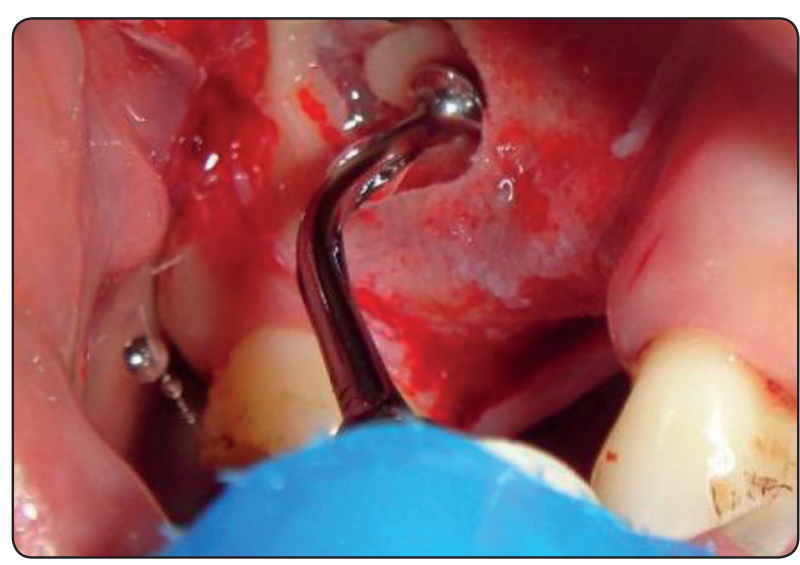

Fig. (2)

- The patients were divided into two groups;

A) PRF group: L- PRF plugs were used to fill the subsinus cavity.

L-PRF Preparation was performed where, at first, $70 \mathrm{ml}$ whole blood was drawn into glass coated plastic tubes without anticoagulant; and was immediately centrifuged at $2000 \mathrm{rpm}$ for 12 minutes. Then, the coagulation cascade resulted from centrifugation led to the formation of a natural fibrin clot in the middle of each tube; with the uppermost layer was represented by the serum (blood plasma without fibrinogen and coagulation factors), and the third layer was a liquid phase containing the white line cells, and stem cells waiting for stimulation and to differentiate into specialized cell types. The lowest third red layer represented platelet rich coagulation consisted of concentrated red and white blood cells, platelets, and clotting factors. The second layer with the fibrin buffy coat and the third liquid phase were removed from the tubes and then used to fill the sinus cavity as shown in figure $(4 a, b)$

Then, the implant was inserted in compression within the residual alveolar bone. The implants were Maxi-Z flat end implants**, with diameter $4.5 \mathrm{~mm}$ and length $10 \mathrm{~mm}$ were placed in the site of

* Variosurg, NSK, Japan.

** Oteocare ${ }^{\mathrm{TM}}$, Implant system, London, UK. 
drilling with the peek carrier as shown in figure (5a). Then, PRF membranes were prepared, where PRF clots were squeezed in their special kit ${ }^{\#}$ to obtain the desired membrane which covered the lateral window of the sinus as shown in figure (5b).

For the xenograft group; where xenograft ${ }^{\S}$ was used to fill the sinus cavity, with simultaneous implant placement in the same way as mentioned above. Then, collagen membrane ${ }^{\ddagger}$ was used to cover the lateral wall of the sinus as shown in figure $(6 a, b)$

\section{VII) Postoperative care and instructions:}

Medications were prescribed, including chlorhexidine rinses twice a day for 14 days, $1 \mathrm{~g}$ amoxicillin two times daily for 7 days., Ibuprofen (400 mg) was taken to control pain. Patients were instructed not to blow their noses for 2 weeks after surgery and to cough or sneeze with an open mouth. They were not allowed to use any removable prosthesis. Sutures were removed 10 days postoperatively.

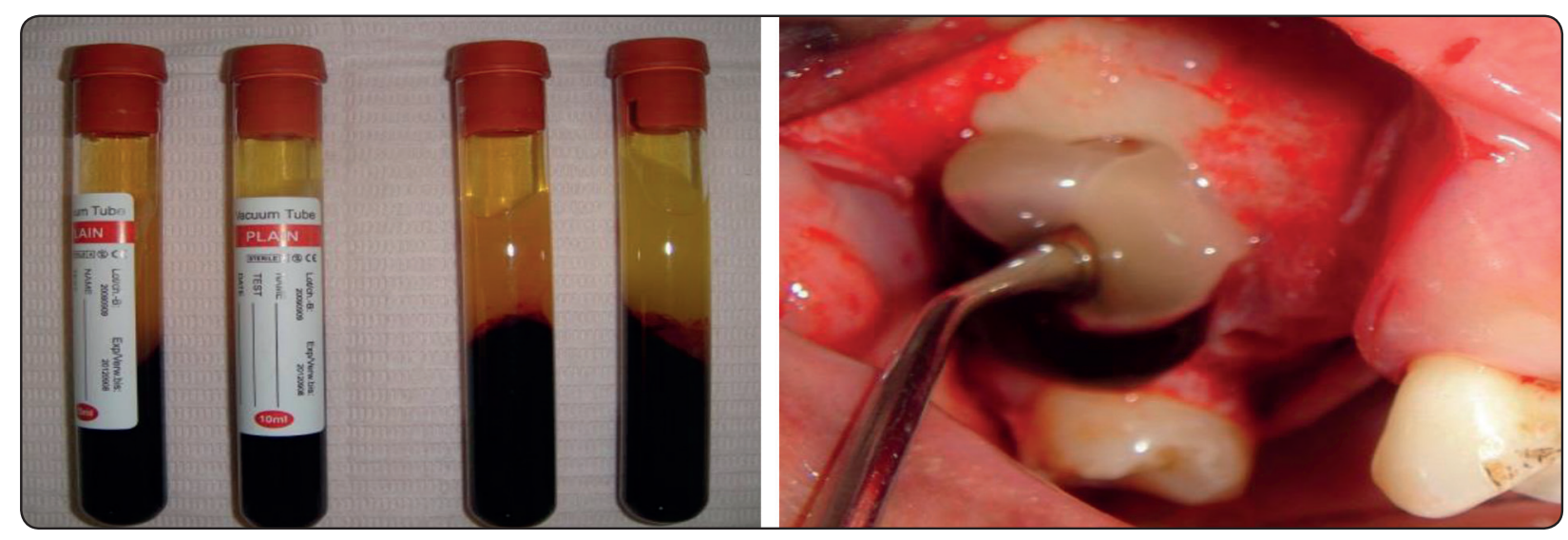

Fig. (4a)

Fig. (4b)

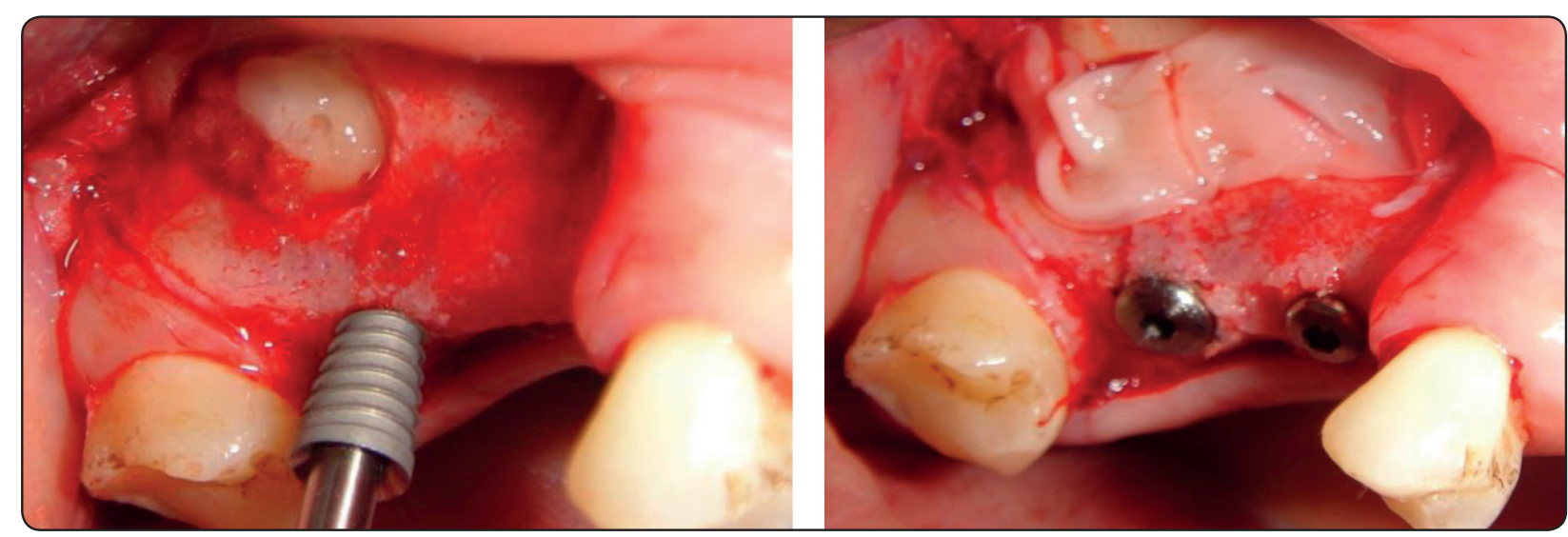

Fig. (5a)

Fig. (5b)

\# Mr. Curette, Korea.

\$ TUTOGEN, RTI I BIOLOGICS TM, Germany.

$¥$ Bioteck, Arcugno, Italy. 


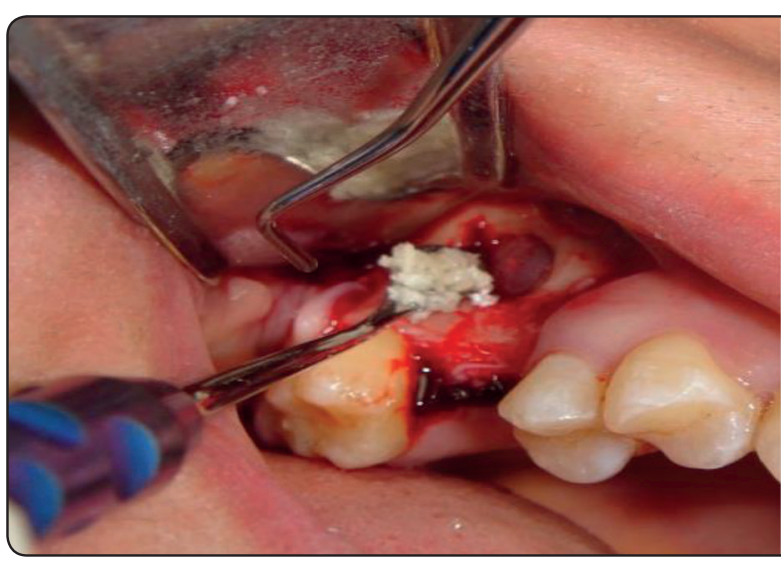

Fig. (6a)

\section{VIII) Follow-up and criteria of clinical evaluation}

Each patient was evaluated at 5 months postoperatively and examined for the following criteria:

- Discomfort, Pain, and Tenderness; as reported according to signs and symptoms of the patients.

\section{IX) Radiographic evaluation:}

Standardized periapical radiographs and CBVT were taken immediately after surgery and at 5 months to detect;

1) Amount of sinus floor elevation.

2) Marginal bone level around the implant.

In CBVT scanning, the raw data set obtained from the CBVT scanning were imported to a special third party software \#\# for secondary reconstruction; and results obtained from the data sets were compared to each other.

For standardization of measurements in CBVT, fusion were used where each image (primary and secondary) were given a color code for identification. The preoperative image was fused to the postoperative image by first using manual registration through anatomical landmarks. Registration (superimposition) was completed automatically by the software allowing the best

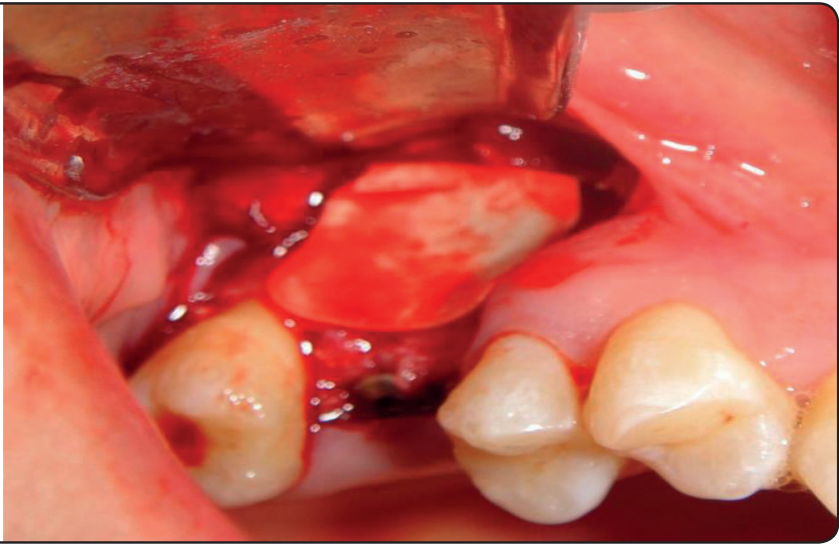

Fig. (6b)

possible accuracy. First measurements were recorded on the primary image. Then the measurement on the primary image was left \& the primary image itself was cancelled leaving the secondary image. A new measurement was recorded on the secondary image on the same plane direction $\&$ cut of the primary image ensuring standardization.

Regarding the prosthetic phase; after a healing period of 5 months, manual punching was performed to expose the implant and a healing collar was placed. After one week, impressions were taken.Then, after construction of the crown in the laboratory, abutments of accurate diameter and angulation were attached to the implants. After that, ceramometallic crown was delivered and cemented after being checked for shade matching, marginal fitness and occlusion as shown in figure (7).

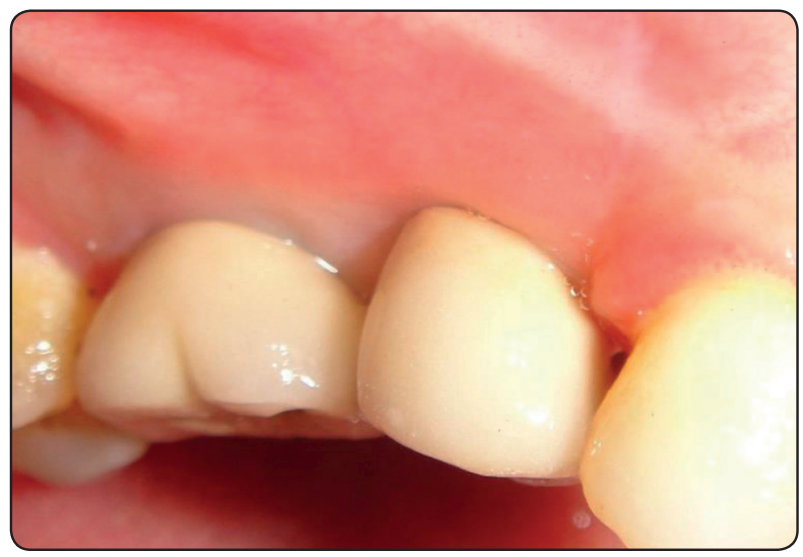

Fig. (7) 


\section{RESULTS}

The study was conducted on 20 patients, 8 males and 12 females with mean age of $(30.5+2.15)$; where they all had single or multiple missing teeth in the maxillary posterior region, with at least one site indicated for implant placement with lateral sinus floor augmentation. The patients were randomly divided into two groups; the first group received PRF as grafting material with PRF membrane covering the lateral window. While the other group received xenograft as a grafting material with collagen membrane covering the lateral window. All patients were followed up for 5 months. Small membrane perforations occurred in two cases without affecting any of the outcomes. All the implants were successfully osseointegrated. Complete soft tissue healing was generally uneventful in all patients after implants placement. No major complications occurred in any of the cases. The results were revealed by clinical and radiographic evaluation.

\section{Clinical evaluation}

It was reported by the patients that swelling and edema in PRF group were less than those in xenograft group.

\section{Statistical analysis}

Based on the clinical and radiographic findings obtained, statistical analyses for all determinations were carried out including the calculation of the mean, standard deviation, and t-value at level $\mathrm{p}<0.05$, for both the groups, control group (Xenograft) \& test group (PRF). The results were statistically evaluated according to the " $\mathrm{t}$ " test.

It was found that the mean crestal bone loss in the PRF group was less than that in the xenograft group after 5 months, being $0.38 \mathrm{~mm} \pm 0.098 \mathrm{SD}$ in $\mathrm{PRF}$ group versus $1.193 \mathrm{~mm} \pm 0.2 \mathrm{SD}$ in xenograft group. There was high statistically significant difference between the two groups at p- value 0.004 as shown in figure (8)

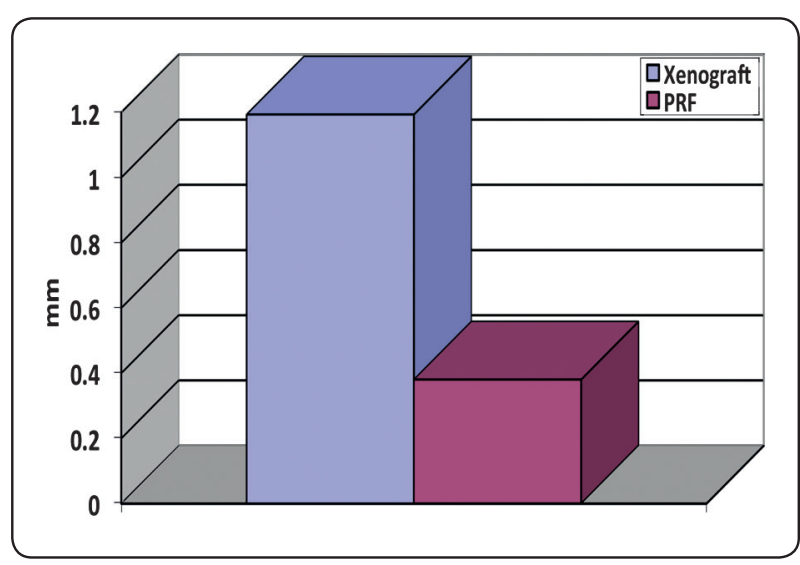

Fig. (8)

\section{DISCUSSION}

Several regenerative techniques were introduced to increase the volume of alveolar bone and improve dimensions of the residual bone height to allow implant placement (Buser et al. 1993). Therefore, sinus floor augmentation as one of these regenerative techniques was introduced to increase the vertical bone height $(\mathrm{VBH})$ to permit placement of longer implants (Jurisic et al. 2008).

Noteworthy, lateral approach in sinus floor augmentation can be performed in one stage with simultaneous implant placement; when the subsinus height is not less than $4 \mathrm{~mm}$ to ensure primary stability. While the 2 stage approach; with delayed implant placement is performed when the sub sinus height less than $4 \mathrm{~mm}$. Therefore, Simultaneous implant placement was used in the present study, as the subsinus heights in all patients were from $4-5 \mathrm{~mm}$; thus allowing primary stability. This was in accordance to Hallman et al. 2001, where it was found that primary stability would be affected when the vertical bone height was less than $4 \mathrm{~mm}$; and increasing the vertical dimension first before implant placement in a second stage would be needed.

Several grafting materials for sinus floor augmentation had been introduced in the clinical practice. These materials are autogenous bone 
grafts, allografts, alloplasts and xenografts (Artzi et al. 2008). One of the main features of xenograft, is its progressive osseointegration with formation of new bone without any signs of acute or chronic inflammation (Traini et al. 2007). Additionally, some authors discovered that bovine bone granules become surrounded by newly formed bone that is first of lamellar type and subsequently undergoes remodeling to become mature bone (Artzi et al. 2005; Orsini et al. 2007). Therefore, in the present study, xenograft was considered the gold standard and used in group I as a comparative group.

In the present study, Leukocyte- platelet- rich fibrin (L-PRF) as a filling material was used in group II as an alternative to commonly used bone grafts. LPRF is a second generation platelet concentrate which is simple and more economic to prepare, as well as less risky to the patients. Moreover, PRF seems to play an important role in angiogenesis via formation of new blood vessels. In addition to the VEGF that present in PRF, which plays a crucial role in the healing process through modulation of angiogenesis and osteogenesis (Wang et al. 2011). Accordingly, the aim of the present study was conducted to compare the effect of platelet-rich fibrin as an alternative to bone graft in lateral sinus floor augmentation versus xenograft in combination with simultaneous implant placement.

For standardization of measurements in CBVT later on, fusion (superimposition program) was used; where each image (primary and secondary) was given a color code for identification. Noteworthy, there were no studies applied CBVT superimpositions in sinus floor augmentation procedures. Therefore, our present study was the first one to apply this technique for standardization of the measurements based on studies justifying the concept of CBVT superimposition (fusion). This was in accordance to (Lagravère et al. 2008), where reference points were registered on two volumetric images. These points were made to coincide when superimposing the two images from different time points. Then, automatic superimposition (so called fusion) was performed to minimize the errors that may be related to the operator's skills.

Regarding the surgical procedure, in the present study, piezoelectric device was used in preparing the lateral window of the maxillary sinus for gaining access to the sinus. It is considered to be conservative and safe in cutting in comparison to other conventional techniques, preserving the integrity of the sinus membrane which is important factor in success of sinus floor elevation technique. This was in accordance to several studies that demonstrated the safety of this technique offers with regard to the proximity of soft tissues such as nerves, vessels, and mucosa. This characteristic was due to low frequency of the ultrasonic waves and the shape of the tip used. In addition to, less thermal damage and maintenance of greater cellular viability (Fathima et al. 2014, Vercellotti 2004;

\section{Gruber et al. 2005).}

Regarding the area of the lateral window, shields or membranes had been used to apply the concept of guided bone regeneration by excluding the undesired cells; to inhibit soft tissue infiltration and thus facilitate healing of the sinus bone graft and help bone recovery. In the xenograft group, collagen membrane was used as a gold standard; according to studies showed the beneficial characteristics of this type of membranes (McAllister et al. 1998, Marinucci et al. 2001). In the L-PRF group, L-PRF membrane was used in comparison due to the above mentioned characteristics of L-PRF such as, mitogenic properties for osteoblastic cells. This was in accordance to some studies comparing L- PRF membrane and conventional collagen membrane. It was revealed that L-PRF seems to be more suitable than collagen membrane for in vitro cultivation of periosteal cells and thus may support the healing process in vivo. This is because the abundance of cytokines present in PRF seemed to 
function as drug delivery system having profound effects on cell development and the composition of the extracellular matrix (Dohan Ehrenfest, Rasmusson, et al. 2009, Gassling et al. 2010).

In our present study, crestal bone loss in L-PRF group was found to be less than that in xenograft group. This finding was considered one of the criteria for evaluating the success rate of implants. This was in agreement with a study reported by Kim et al. 2009, where implant success rate was evaluated after sinus floor augmentation with xenograft and simultaneous implant placement. Cases with crestal bone loss $\leq 1.5 \mathrm{~mm}$ were considered to be successful. These results were comparable to our study with $0.38 \mathrm{~mm}$ crestal bone loss in L- PRF group versus $1.19 \mathrm{~mm}$ in xenograft group.

Therefore, Findings obtained from the present study, revealed that applying L-PRF in sinus floor augmentation can yield better results. Also, it seems to replace the expensive xenograft material and collagen membrane.

\section{CONCLUSION}

- L-PRF group showed superior results over the xenograft group; where crestal bone loss in PRF group was less than that in xenograft group.

- L-PRF can be used as a grafting material and as a membrane.

\section{REFERENCES}

1. Artzi, Z. et al., 2008. Histomorphometric assessment of bone formation in sinus augmentation utilizing a combination of autogenous and hydroxyapatite/biphasic tricalcium phosphate graft materials: at 6 and 9 months in humans. Clinical oral implants research, 19(7), pp.686-92. Available at: http://www.ncbi.nlm.nih.gov/ pubmed/18492077 [Accessed August 10, 2014].

2. Artzi, Z. et al., 2005. The amount of newly formed bone in sinus grafting procedures depends on tissue depth as well as the type and residual amount of the grafted material. Journal of clinical periodontology, 32(2), pp.193-9. Available at: http://www.ncbi.nlm.nih.gov/ pubmed/15691351 [Accessed December 6, 2014].

3. Boyne, P.J. \& James, R.A., 1980. Grafting of the maxillary sinus floor with autogenous marrow and bone. Journal of oral surgery (American Dental Association : 1965), 38(8), pp.613-6. Available at: http://www.ncbi.nlm.nih.gov/ pubmed/6993637 [Accessed September 7, 2014].

4. Buser, D. et al., 1993. Localized ridge augmentation using guided bone regeneration. 1. Surgical procedure in the maxilla. The International journal of periodontics \& restorative dentistry, 13(1), pp.29-45. Available at: http://www.ncbi.nlm.nih.gov/pubmed/8330945 [Accessed November 28, 2014].

5. Chanavaz, M., 1990. Maxillary sinus: anatomy, physiology, surgery, and bone grafting related to implantology--eleven years of surgical experience (1979-1990). The Journal of oral implantology, 16(3), pp.199-209. Available at: http://www.ncbi.nlm.nih.gov/pubmed/2098563 [Accessed August 17, 2014].

6. Diss, A. et al., 2008. Osteotome sinus floor elevation using Choukroun's platelet-rich fibrin as grafting material: a 1-year prospective pilot study with microthreaded implants. Oral Surgery, Oral Medicine, Oral Pathology, Oral Radiology, and Endodontology, 105(5), pp.572-579.

7. Dohan Ehrenfest, D.M., de Peppo, G.M., et al., 2009. Slow release of growth factors and thrombospondin-1 in Choukroun's platelet-rich fibrin (PRF): a gold standard to achieve for all surgical platelet concentrates technologies. Growth factors (Chur, Switzerland), 27(1), pp.63-9. Available at: http://www.ncbi.nlm.nih.gov/ pubmed/19089687 [Accessed December 7, 2014].

8. Dohan Ehrenfest, D.M., Rasmusson, L. \& Albrektsson, T., 2009. Classification of platelet concentrates: from pure platelet-rich plasma (P-PRP) to leucocyte-and plateletrich fibrin (L-PRF). Trends in biotechnology, 27(3), pp.158-167.

9. Fathima, K.H. et al., 2014. Perforated schneiderian membrane repair during sinus augmentation in conjunction with immediate implant placement. Journal of pharmacy \& bioallied sciences, 6(Suppl 1), pp.S174-7. Available at: http://www.pubmedcentral.nih.gov/articlerender.fcgi ?artid $=4157262 \&$ tool=pmcentrez\&rendertype $=$ abstract [Accessed October 16, 2014].

10. Gassling, V. et al., 2010. Platelet-rich fibrin membranes as scaffolds for periosteal tissue engineering. Clinical oral 
implants research, 21(5), pp.543-9. Available at: http:// www.ncbi.nlm.nih.gov/pubmed/20443805 [Accessed November 27, 2014].

11. Gruber, R. et al., 2003. Platelets are mitogenic for periosteum-derived cells. Journal of orthopaedic research : official publication of the Orthopaedic Research Society, 21(5), pp.941-8. Available at: http://www.ncbi.nlm.nih. gov/pubmed/12919885 [Accessed December 5, 2014].

12. Gruber, R.M. et al., 2005. Ultrasonic surgery--an alternative way in orthognathic surgery of the mandible. A pilot study. International journal of oral and maxillofacial surgery, 34(6), pp.590-3. Available at: http://www.ncbi.nlm. nih.gov/pubmed/16053884 [Accessed December 3, 2014].

13. Holtzclaw, D. et al., 2008. The safety of bone allografts used in dentistry: a review. Journal of the American Dental Association (1939), 139(9), pp.1192-9. Available at: http:// www.ncbi.nlm.nih.gov/pubmed/18762629 [Accessed September 26, 2014].

14. Jurisic, M. et al., 2008. Maxillary sinus floor augmentation: comparing osteotome with lateral window immediate and delayed implant placements. An interim report. Oral surgery, oral medicine, oral pathology, oral radiology, and endodontics, 106(6), pp.820-7. Available at: http://www. ncbi.nlm.nih.gov/pubmed/18602304 [Accessed November 28, 2014].

15. Kaigler, D., Silva, E.A. \& Mooney, D.J., 2013. Guided bone regeneration using injectable vascular endothelial growth factor delivery gel. Journal of periodontology, 84(2), pp.230-8. Available at: http://www.pubmedcentral. nih.gov/articlerender.fcgi?artid=3669541\&tool=pmcentre z\&rendertype=abstract [Accessed December 5, 2014].

16. Kim, Y.-K. et al., 2009. Evaluation of sinus bone resorption and marginal bone loss after sinus bone grafting and implant placement. Oral surgery, oral medicine, oral pathology, oral radiology, and endodontics, 107(2), pp.e21-8. Available at: http://www.ncbi.nlm.nih.gov/ pubmed/19138634 [Accessed August 10, 2014].

17. Lagravère, M.O. et al., 2008. Three-dimensional accuracy of measurements made with software on cone-beam computed tomography images. American journal of orthodontics and dentofacial orthopedics : official publication of the American Association of Orthodontists, its constituent societies, and the American Board of Orthodontics, 134(1), pp.112-6. Available at: http://www.ncbi.nlm.nih. gov/pubmed/18617110 [Accessed December 6, 2014].
18. Lundgren, S. et al., 2004. Bone reformation with sinus membrane elevation: a new surgical technique for maxillary sinus floor augmentation. Clinical implant dentistry and related research, 6(3), pp.165-73. Available at: http://www.ncbi.nlm.nih.gov/pubmed/15726851.

19. Marinucci, L. et al., 2001. In vitro comparison of bioabsorbable and non-resorbable membranes in bone regeneration. Journal of periodontology, 72(6), pp.753-9. Available at: http://www.ncbi.nlm.nih.gov/ pubmed/11453237 [Accessed December 7, 2014].

20. Mazor, Z. et al., 2009. Sinus floor augmentation with simultaneous implant placement using Choukroun's platelet-rich fibrin as the sole grafting material: a radiologic and histologic study at 6 months. Journal of periodontology, 80(12), pp.2056-2064.

21. McAllister, B.S. et al., 1998. Residual lateral wall defects following sinus grafting with recombinant human osteogenic protein-1 or Bio-Oss in the chimpanzee. The International journal of periodontics \& restorative dentistry, 18(3), pp.227-39. Available at: http://www.ncbi.nlm.nih. gov/pubmed/9728105 [Accessed December 7, 2014].

22. Metzler, P. et al., 2012. The effect of current used bone substitution materials and platelet-rich plasma on periosteal cells by ectopic site implantation: an in-vivo pilot study. Journal of cranio-maxillo-facial surgery: official publication of the European Association for CranioMaxillo-Facial Surgery, 40(5), pp.409-15. Available at: http://www.ncbi.nlm.nih.gov/pubmed/21872487 [Accessed December 5, 2014].

23. MW, M., 2005. Fibrinogen and fibrin structure and functions. J Thromb Haemost, 3, p.1894e-1904.

24. Nasr, H.F., Aichelmann-Reidy, M.E. \& Yukna, R.A., 1999. Bone and bone substitutes. Periodontology 2000, 19, pp.74-86. Available at: http://www.ncbi.nlm.nih.gov/ pubmed/10321217 [Accessed September 24, 2014].

25. Ning, Y. et al., 2009. The research of degradability of a novel biodegradable coralline hydroxyapatite after implanted into rabbit. Journal of biomedical materials research. Part A, 88(3), pp.741-6. Available at: http://www.ncbi.nlm.nih. gov/pubmed/18357581 [Accessed September 28, 2014].

26. Orsini, G. et al., 2007. Histological and ultrastructural evaluation of bone around Bio-Oss particles in sinus augmentation. Oral diseases, 13(6), pp.586-93. Available at: http://www.ncbi.nlm.nih.gov/pubmed/17944677 [Accessed December 6, 2014]. 
27. Peleg, M., Garg, A.K. \& Mazor, Z., 2006. Predictability of simultaneous implant placement in the severely atrophic posterior maxilla: A 9-year longitudinal experience study of 2132 implants placed into 731 human sinus grafts. The International journal of oral \& maxillofacial implants, 21(1), pp.94-102. Available at: http://www.ncbi.nlm.nih. gov/pubmed/16519187.

28. Reynolds, M.A., Aichelmann-Reidy, M.E. \& BranchMays, G.L., 2010. Regeneration of periodontal tissue: bone replacement grafts. Dental clinics of North America, 54(1), pp.55-71. Available at: http://www.ncbi.nlm.nih. gov/pubmed/20103472 [Accessed September 26, 2014].

29. Rosenberg, E. \& Rose, L.F., 1998. Biologic and clinical considerations for autografts and allografts in periodontal regeneration therapy. Dental clinics of North America, 42(3), pp.467-90. Available at: http://www.ncbi.nlm.nih. gov/pubmed/9700450 [Accessed September 24, 2014].

30. Sohn, D.-S. et al., 2010. New bone formation in the maxillary sinus using only absorbable gelatin sponge. Journal of oral and maxillofacial surgery : official journal of the American Association of Oral and Maxillofacial Surgeons, 68(6), pp.1327-33. Available at: http://www. ncbi.nlm.nih.gov/pubmed/20493382 [Accessed August 10, 2014].

31. Summers, R.B., 1994. The osteotome technique: Part 3--Less invasive methods of elevating the sinus floor. Compendium (Newtown, Pa.), 15(6), pp.698-700.

32. Tatum, H., 1986. Maxillary and sinus implant reconstructions. Dental clinics of North America, 30(2), pp.207-29. Available at: http://www.ncbi.nlm.nih.gov/ pubmed/3516738 [Accessed December 5, 2014].

33. Traini, T. et al., 2007. A histologic and histomorphometric evaluation of anorganic bovine bone retrieved 9 years after a sinus augmentation procedure. Journal of periodontology, 78(5), pp.955-61. Available at: http://www.ncbi.nlm.nih. gov/pubmed/17470032 [Accessed December 6, 2014].

34. Vercellotti, T., 2004. Technological characteristics and clinical indications of piezoelectric bone surgery. Minerva stomatologica, 53(5), pp.207-14. Available at: http://www. ncbi.nlm.nih.gov/pubmed/15263877 [Accessed December 5, 2014].

35. Wang, C.-J. et al., 2011. VEGF modulates angiogenesis and osteogenesis in shockwave-promoted fracture healing in rabbits. The Journal of surgical research, 171(1), pp.114-9. Available at: http://www.ncbi.nlm.nih.gov/ pubmed/20452608 [Accessed December 6, 2014].

36. Wenz, B., Oesch, B.\& Horst, M., 2001. Analysis of the risk of transmitting bovine spongiform encephalopathy through bone grafts derived from bovine bone. Biomaterials, 22(12), pp.1599-606. Available at: http://www.ncbi.nlm. nih.gov/pubmed/11374460 [Accessed September 28, 2014].

37. Wood,R.M. \& Moore,D.L., 1988. Grafting of the maxillary sinus with intraorally harvested autogenous bone prior to implant placement. The International journal of oral \& maxillofacial implants, 3(3), pp.209-14. Available at: http://www.ncbi.nlm.nih.gov/pubmed/3074053 [Accessed September 7, 2014]. 